\title{
Análisis de la influencia del tratamiento térmico de envejecimiento en la modificación de las propiedades mecánicas de la aleación AA6060 procesada por $\operatorname{ECAE}^{(\cdot)}$
}

\author{
I. Pérez*, C. Luis-Pérez*, R. Luri*, J. León* e I. Puertas*
}

\begin{abstract}
Resumen
En el presente trabajo se ha realizado un estudio de la modificación de las propiedades mecánicas de la aleación de aluminio AA6060 F, al ser deformada mediante deformación plástica severa empleando el proceso de extrusión en canal angular (ECAE). Asimismo, se ha efectuado un estudio del efecto del envejecimiento artificial sobre la modificación de dichas propiedades mecánicas. Para ello, se han realizado tratamientos térmicos a diferentes temperaturas y tiempos de permanencia, efectuando mediciones de dureza, ensayos de tracción, ensayos con péndulo Charpy y técnicas de revelado metalográfico, para conocer los comportamientos mecánicos y el estado del material. Existe un elevado número de publicaciones, que han analizado el efecto de tratamientos térmicos de envejecimiento en aleaciones de la serie 6000 . Sin embargo, la aleación AA6060 no ha sido tan ampliamente estudiada. Por ello, en este trabajo se parte de una aleación comercial AA6060 en estado de bruto de colada, lo cual no ha sido estudiado hasta la fecha.
\end{abstract}

\section{Analysis of the influence of aging heat treatment on the modification of the mechanical properties of the alloy AA6060 processed by ECAE}

\begin{abstract}
In the present work, a study on the modification of the mechanical properties of the aluminium alloy AA6060 F has been made when severe plastic deformation is applied to it through Equal Channel Angular Extrusion (ECAE). Moreover, a study about the effect of the artificial aging on the modification of such mechanical properties has been carried out after having processed the material by ECAE. In order to do this, thermal treatments at different values of temperature and holding time have been performed. Hardness, tensile tests and Charpy impact test, along with metallographic techniques, have been carried out in order to analyse the mechanical behaviour and the state of the material. In spite of the existence of several studies that have analysed the effect of aging thermal treatments on 6000 aluminium alloys, such studies are focused on laboratory alloys with industrial compositions not very usual. Therefore, in the present work, the employed starting material is a commercial alloy as-cast, which has not been studied up to now.
\end{abstract}

Keywords $\quad$ ECAP; SPD; Aging; Aluminium; UFG

\section{INTRODUCCIÓN}

Actualmente, hay un gran interés en la producción de materiales de grano ultrafino (UFG), tanto para fines de investigación, como comerciales. Una de las técnicas que recientemente ha despertado mayor interés para la obtención de materiales de grano ultrafino, es la deformación plástica severa (SPD). Estudios como el realizado por M. Furukawa ${ }^{[1]}$ describen la generación de materiales con tamaños de grano en el rango micrométrico e incluso nanométrico mediante la aplicación de deformación plástica severa.

En el trabajo de revisión bibliográfica realizado por Valiev et al. ${ }^{[2]}$ se describen diferentes técnicas para aplicar altos grados de deformación, tales como: ECAE, HPT y ARB, entre otras, siendo la deformación plástica severa (SPD) un método favorable para conformar y controlar los comportamientos mecánicos de los materiales.

(•) Trabajo recibido el día 15 de Febrero de 2010 y aceptado en su forma final el día 17 de Agosto de 2010.

* Departamento de Ingeniería Mecánica, Energética y de Materiales, Universidad Pública de Navarra, España. 
En el presente trabajo, se emplea el proceso denominado como extrusión en canal angular constante (ECAE), que consiste en un proceso de deformación plástica severa (SPD), ideado en 1972 por V.M. Segal et al. ${ }^{[3]}$ en la antigua Unión Soviética.

El proceso ECAE, tal y como se muestra en estudios como el de González et al. ${ }^{[4]}$, consiste en realizar una extrusión a través de una matriz que tiene dos canales de igual sección transversal, que se intersectan en un ángulo comprendido, generalmente, entre $90^{\circ}$ y $135^{\circ}$. Al extruir el material, éste atraviesa la intersección entre los canales, siendo deformado por tensión cortante en presencia de una elevada presión hidrostática, impuesta por la restricción que impone la propia matriz de ECAE, lo cual evita su rotura y permite que pueda deformarse hasta valores de deformación, muy superiores a los que es posible obtener con procesos termomecánicos convencionales.

Existen varios trabajos que centran su estudio en analizar la modificación de propiedades mecánicas que aporta la generación de este tipo de microestructuras. Concretamente, en el artículo publicado por Hockauf ${ }^{[5]}$ se analizan las mejoras en la resistencia mecánica y la ductilidad de la AA6060 procesada por ECAE. Otros trabajos tales como el de M.J. O'Brien ${ }^{[6]}$, evalúan comportamientos superplásticos proporcionados por el afino de grano. En el trabajo de Agena ${ }^{[7]}$, se muestra la modificación de las propiedades mecánicas de la AA6082 al ser procesada mediante SPD.

$\mathrm{Al}$ igual que en otras aleaciones, se han realizado multitud de ensayos para evaluar la modificación de propiedades que realiza el ECAE sobre aluminios que pertenecen a la familia 6000 . Se ha estudiado el afino de grano obtenido tras realizar tratamientos adecuados de recristalización en probetas previamente procesadas, obteniéndose granos submicrométricos $(0,5 \mu \mathrm{m})$, mejorándose la capacidad de fluencia del material procesado y proporcionando un ahorro de energía al poder reducirse la temperatura necesaria para realizar la forja, según se muestra en el trabajo de Chaudhury ${ }^{[8]}$.

El proceso de ECAE se ha aplicado a un gran número de materiales. En el trabajo de S.N. Mathaudhu ${ }^{[9]}$, se muestran los resultados obtenidos tras procesar en caliente muestras de wolframio mediante ECAE. En dicho trabajo se obtuvieron granos de menor tamaño al aumentar el número de pasadas (desde 1 hasta 4) y al disminuir la temperatura de trabajo (desde $1.200^{\circ} \mathrm{C}$ hasta $1.000^{\circ} \mathrm{C}$ ), obteniéndose granos de $350 \mathrm{~nm}$ al procesar las probetas cuatro veces a $1.000^{\circ} \mathrm{C}$. El cobre también ha sido analizado en estudios como el de N. Lugo ${ }^{[10]}$ que procesó muestras hasta 16 pasadas, observando que las principales modificaciones en las propiedades mecánicas y el mayor afino de grano se producía en la pri- mera pasada siendo más moderado en las siguientes pasadas de ECAE.

En lo que respecta a las aleaciones de aluminio, cabe destacar la serie 6.000 que son aleaciones tratables térmicamente. Los tratamientos térmicos pueden ser estáticos o dinámicos, naturales o artificiales y consisten en la precipitación de compuestos intermedios, por difusión de aleantes que se encuentran disueltos en la matriz. Aspectos como la cinética de precipitación de estos aleantes o los mecanismos implicados en la misma, se tratan de elucidar en artículos como el de Roven ${ }^{[11]}$, justificándose los picos de dureza en función de la morfología que presentan los compuestos precipitados. El papel que desempeñan estas segundas fases, capaces de endurecer el material por medio del bloqueo de los sistemas de deslizamiento o proporcionando estabilidad térmica al fijar las juntas de grano evitando su crecimiento, según relata Ravi Shankar ${ }^{[12]}$, son aspectos interesantes en el comportamiento de esta familia de aleaciones.

Las distintas combinaciones existentes entre el procesado por ECAE y el tratamiento previo o posterior de precipitación, son tratadas en estudios como el de Kim y Jeong ${ }^{[13]}$ o el de Kim y Wang ${ }^{[14]}$, que justifican siempre que el tratamiento de precipitación se realice tras el procesado de las probetas.

En el presente artículo, se analizarán algunos de los aspectos nombrados anteriormente. Para ello, se utiliza la aleación AA6060 F que se encuentra en estado comercial, sin haber recibido ningún tratamiento de solución ni de homogenización, lo cual conlleva un ahorro en el coste de fabricación. Este estudio no ha sido realizado con anterioridad según se desprende de la bibliografía consultada, con lo cual, presenta interés desde el punto de vista de avanzar en el conocimiento del efecto de aplicar una deformación plástica severa, en aleaciones de aluminio tratables térmicamente. Se prestará especial atención a la modificación de propiedades obtenidas tras el procesado por ECAE, obteniéndose una gráfica de endurecimiento en función del número de pasadas efectuadas, que van desde NO hasta N16.

Asimismo, se representará el comportamiento mostrado por el material deformado y sin deformar, al aplicarse tratamientos de precipitación a tres temperaturas distintas, tratándose de determinar las combinaciones de temperatura y tiempo óptimas para conseguir las propiedades más beneficiosas para su posterior uso.

\section{PROCEDIMIENTO EXPERIMENTAL}

La tabla I muestra la composición química de la AA6060 analizada por medio de espectrofotometría 
Tabla I. Composición química de la aleación AA6060 realizada por ICP

Table I. Chemical composition of AA6060 alloy performed by ICP

\begin{tabular}{cccccc}
\hline Elemento & Al & Si & Mg & Fe & Mn \\
\hline$\%(p / p)$ & $98,36 \pm 0,60$ & $43 \pm 0,006$ & 0,366 & 0,24 & 0,04 \\
\hline Elemento & $\mathbf{T i}$ & $\mathbf{Z n}$ & $\mathbf{C r}$ & $\mathbf{C u}$ \\
\hline$\%(p / p)$ & 0,03 & 0,02 & 0,006 & 0,002 \\
\hline
\end{tabular}

de emisión óptica por plasma acoplado inductivamente (ICP)-Radial. El aluminio fue cuantificado con independencia del resto de elementos siguiéndose el mismo procedimiento con el silicio, obteniéndose con un intervalo de confianza correspondiente al $95 \%( \pm 0,5)$.

En la tabla I, se muestran los resultados obtenidos por el ICP en los que se determinaron cantidades de $\mathrm{Al}, \mathrm{Si}, \mathrm{Mg}, \mathrm{Cr}, \mathrm{Cu}, \mathrm{Fe}, \mathrm{Mn}, \mathrm{Ti}$ y Zn.

Tal y como era de esperar, el contenido en aluminio, el cual forma la fase primaria, se encuentra cercano al 98 \%. Los componentes mayoritarios que forman las segundas fases son el silicio y el magnesio. El hierro también es una impureza proveniente del propio mineral del que se extrae el aluminio por electrólisis de forma industrial. Dicho elemento forma, junto al propio aluminio y al silicio, precipitaciones de $\mathrm{AlFeSi}$, que suelen depositarse en las fronteras de grano. La composición del material esta dentro de los márgenes establecidos por la norma UNE 38337:2001 ${ }^{[15]}$.

El material, que se encuentra en bruto de colada, es mecanizado en forma de probetas de $80 \mathrm{~mm}$ de longitud y de sección cuadrada de $9 \mathrm{~mm}$ x $9 \mathrm{~mm}$, tal y como se muestra en la figura 1. Esta operación es necesaria para que puedan ser procesadas por ECAE ya que son las dimensiones de las matrices utilizadas

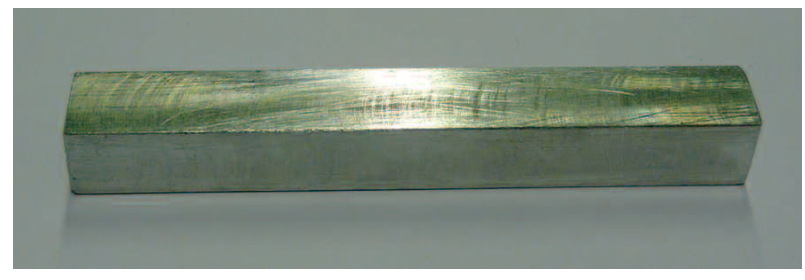

Figura 1. Material de inicio (N0).

Figure 1. Starting material (NO). para aplicar la deformación, tal y como puede observarse en la figura 2.

En la figura 2 se muestran las matrices empleadas en los experimentos. Dichas matrices han sido fabricadas con radios iguales de 1,95 mm y un ángulo entre canales de $\phi=90^{\circ}$, siguiendo las recomendaciones mostradas en C.J. Luis ${ }^{[16]}$ para el diseño de las matrices de extrusión en canal angular.

En el trabajo realizado por R. Luri y C.J. Luis ${ }^{[17]}$ se desarrollaron una serie de expresiones analíticas para calcular la deformación plástica impartida al material procesado por ECAE. Para el caso de que $\mathrm{R}_{\text {ext }} \geq \mathrm{R}_{\text {int }}$, lo cual corresponde a la situación del ECAE tradicional, la deformación equivalente puede calcularse a partir de la ecuación (1), siendo $\bar{\varepsilon}$ la deformación equivalente, $\mathrm{D}$ la longitud de la arista de la matriz de sección cuadrada, $\phi$ el ángulo interno de la matriz y $\Psi$ el ángulo exterior de la matriz, tal y

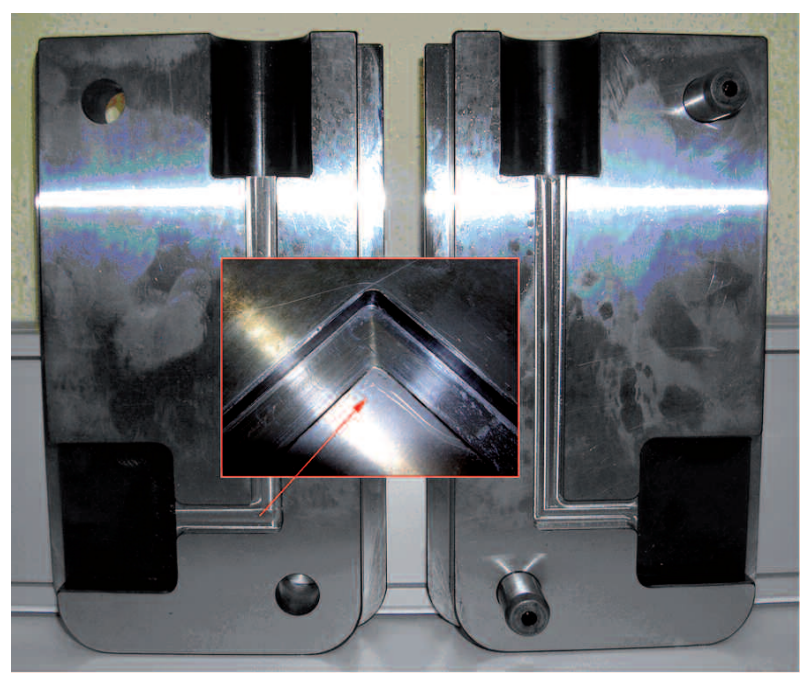

Figura 2. Matrices utilizadas para el proceso de ECAE.

Figure 2. Dies used in ECAE process. 
como puede observarse en la figura $3^{[18]}$, que se puede calcular a partir de la ecuación (2).

$$
\begin{aligned}
& \bar{\varepsilon}=\frac{2 \cot \left(\frac{\Phi}{2}+\frac{\Psi}{2}\right)+(\pi-\Phi)\left[1-\cot \left(\frac{\Phi}{2}+\frac{\Psi}{2}\right) \tan \left(\frac{\Phi}{2}\right)\right]}{\sqrt{3}} \\
& \Psi=2 \tan ^{-1}\left[\frac{\left(\frac{R_{e x t}}{D}-\frac{R_{\text {int }}}{D}\right) \tan \left(\frac{\Phi}{2}\right)}{1+\frac{R_{\text {int }}}{D}-\frac{R_{e x t}}{D}+\tan ^{2}\left(\frac{\Phi}{2}\right)}\right]
\end{aligned}
$$

Como se ha indicado anteriormente, en el presente trabajo se han procesado por ECAE piezas de $80 \mathrm{~mm}$ de longitud y con sección cuadrada de $9 \mathrm{~mm} \times 9 \mathrm{~mm}$, tal y como se muestra en la figura 4 . La geometría de la matriz empleada es con $\phi=90^{\circ}$, $\mathrm{R}_{\text {int }}=\mathrm{R}_{\text {ext }}=1,95 \mathrm{~mm}$ y $\mathrm{D}=10 \mathrm{~mm}$. Aplicando las ecuaciones (1) y (2), se obtiene que, $\Psi=0^{\circ}$, y por tanto, la deformación media impartida al material en estado N1 es de $\bar{\varepsilon}=1,16$. Se puede considerar que existe efecto aditivo a la hora de calcular la deformación introducida en las siguientes pasadas, por lo tanto, $\left(\bar{\varepsilon}_{f}\right.$, siendo $\mathrm{N} \bar{\varepsilon}$ el número de pasadas), de manera que para probetas en estado $\mathrm{N} 2$ y N4 los valores corresponden a 2,32 y 4,64, respectivamente.

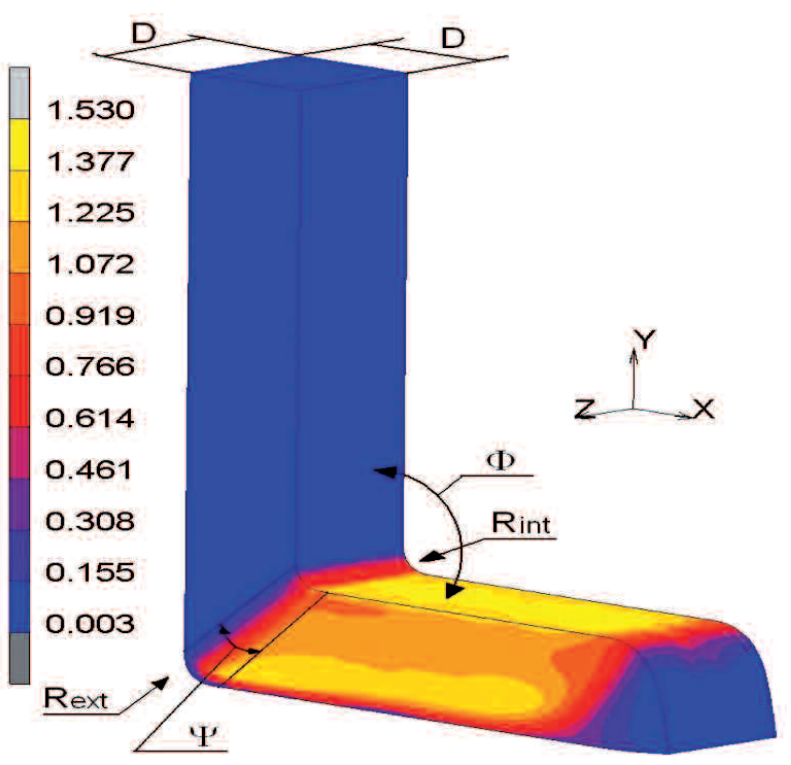

Figura 3. Simulación por M.E.F. del procesado mediante ECAE.

Figure 3. FEM simulation of ECAE process.

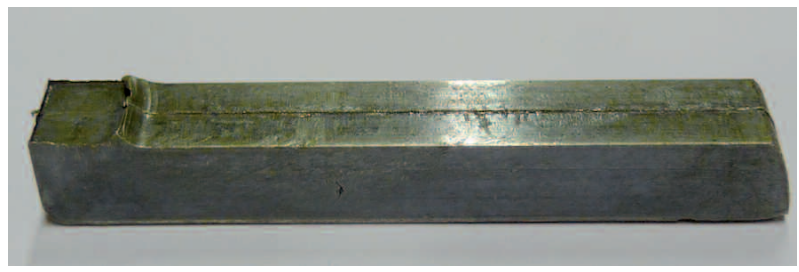

Figura 4. Material procesado 2 veces por ECAE según ruta $C(\mathrm{~N} 2)$.

Figure 4. Material after two ECAE passages according to route $C$ (N2).

En los ensayos se utilizó Ruta C consistente en rotar $180^{\circ}$ las probetas, tras cada pasada, repitiéndose esta secuencia las veces que sea necesario hasta conseguir deformaciones desde N2 hasta N16, realizadas a temperatura ambiente.

Se efectuaron tratamientos térmicos de envejecimiento a tres temperaturas distintas, $125^{\circ} \mathrm{C}, 150^{\circ} \mathrm{C}$ y $175^{\circ} \mathrm{C}$ y con tiempos de calentamiento desde $15 \mathrm{~min}$ hasta $10 \mathrm{~h}$ y, en determinados casos, hasta $75 \mathrm{~h}$, con el fin de abarcar un intervalo amplio de trabajo que pueda representar el comportamiento térmico de la aleación considerada en este trabajo. Las muestras se introdujeron en el horno una vez alcanzada la temperatura de tratamiento, con objeto de eliminar la variabilidad del efecto de la rampa de calentamiento en el presente estudio.

Se realizaron un total de diez indentaciones en cada probeta con el fin de obtener valores promedios de dureza. La carga utilizada fue de 0,3 kg. Se efectuaron ensayos con péndulo Charpy, así como ensayos de tracción por triplicado, con objeto de determinar la resistencia mecánica y el alargamiento a rotura.

\section{ANÁLISIS $Y$ DISCUSIÓN DE RESULTADOS}

En esta sección se discutirá la modificación de propiedades obtenidas, tanto cuando se aplica el proceso de ECAE sin tratamiento térmico, como cuando se combina el proceso de ECAE con tratamientos de envejecimiento posteriores.

\subsection{Análisis de las propiedades mecánicas sin tratamientos térmicos}

El proceso de ECAE en el presente estudio, ha sido realizado a temperatura ambiente, con velocidades de deformación entorno a $0,37 \mathrm{~s}^{-1}$ y velocidades de 
procesado de $50 \mathrm{~mm} / \mathrm{min}$. En estas condiciones, se considera que no existe un aumento de temperatura considerable, por lo que el proceso se desarrolla a temperatura ambiente.

En los procesos de SPD, se produce un mayor afino de grano y una mayor desorientación entre los granos y subgranos de la estrucutura, que en los procesos de deformación plástica convencionales. La nueva red de estructura submicrométrica y/o nanométrica va reemplazando a la anterior ${ }^{[1,19}$ y 20]. Al aplicar tratamientos térmicos, se puede provocar alivios en la red, recristalizaciones y crecimientos de grano.

El proceso de ECAE, en presencia de precipitados, provoca un refinamiento de los mismos mediante fragmentación, según relatan estudios como el de Roven ${ }^{[11]}$.

Se han realizado mediciones de dureza Vickers (diez indentaciones por cada punto representado) en probetas, tanto en estado inicial (NO), como en aquellas procesadas hasta N2, N4, N12, N14 y N16. Los valores de dureza obtenidos han sido analizados junto con su incertidumbre expandida para un intervalo de confianza del $95 \%$, según la ley de propagación de varianzas de la G.U.M. (Guide to the expresión of uncertainty in measurements) ${ }^{[21]}$.

Se ajustan los valores obtenidos a una ecuación tipo Hollomon $\left(H V=k_{0}+k \varepsilon^{n}\right)$ aplicada en este caso a la dureza, obteniéndose una curva de ajuste exponencial de la dureza frente a la deformación real $\varepsilon$. Para ello, se ha tenido en cuenta la expresión mostrada en la ecuación (1) y que $\bar{\varepsilon}_{f}=\mathrm{N} \bar{\varepsilon}$, siendo $\bar{\varepsilon}$ la deformación plástica equivalente en una pasada de ECAE y $\bar{\varepsilon}_{f}$ la deformación plástica que tiene tras $\mathrm{N}$ pasadas.

$\mathrm{Tal}$ y como se aprecia en la figura 5 , se consigue una buena aproximación a los valores experimentales obtenidos, incluyéndose además, la expresión que relaciona la dureza y la deformación real.

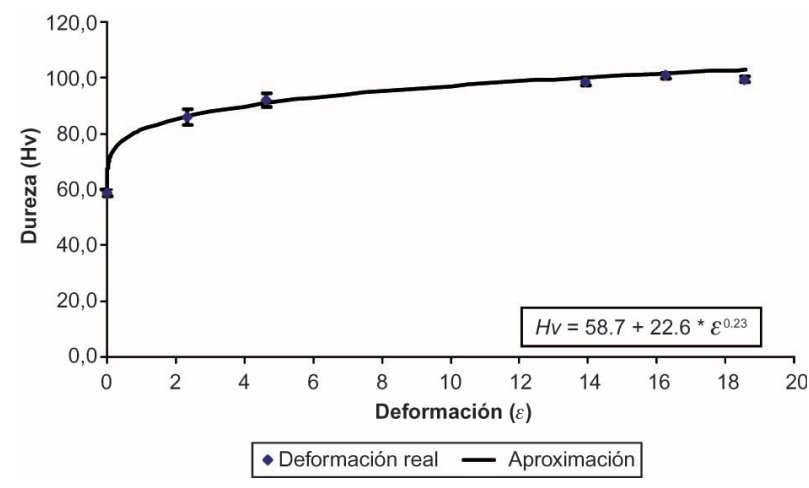

Figura 5. Aproximación exponencial de Deformación real $(\varepsilon)$ con respecto a dureza $(\mathrm{HV})$.

Figure 5. Exponential approach for True strain $(\varepsilon)$ with respect to hardness (HV).
Como se puede apreciar, la dureza registra valores crecientes. A continuación, se cuantifican los incrementos porcentuales de dureza, utilizando la ecuación (3), donde HVf y HVi son los valores finales e iniciales de dureza Vickers, respectivamente.

$$
\frac{H V f-H V i}{H V i} * 100
$$

Tal y como se observa en la tabla II, el material experimenta incrementos significativos en la dureza al aumentar el número de pasadas de ECAE.

La mayor mejora se encuentra en las dos primeras fases del proceso con una pendiente máxima en su primer tramo, dando un valor del $46,3 \%$ al evaluar los valores de N0 y N2. En estados de deformación cercanos a la saturación por dislocaciones correspondientes a N14, la dureza presenta valores próximos a $100 \pm 0,7 \mathrm{HV}$, lo que corresponde a una mejora porcentual del $72 \%$ respecto a NO. Sin embargo, la dureza sólo aumenta un 9,7\% al procesar la aleación por ECAE desde N4 a N14.

En la figura 6 se representan los resultados promedio de resistencia mecánica y de alargamiento a rotura, obtenidos al realizar ensayos de tracción por triplicado sobre probetas de ECAE en N0, N2 y N4. Se empleó para ello una máquina de ensayos tracción de 200 kN con un extensómetro acoplado y se analizaron los resultados junto con su incertidumbre expandida con un intervalo de confianza del $99 \%$, según la ley de propagación de varianzas de la G.U.M. [21].

En la figura 6 se puede apreciar el aumento en la resistencia máxima a tracción $(\mathrm{Rm})$ que proporciona el procesado por ECAE. Se vuelve a observar, que el principal aumento se produce en las dos primeras pasadas (92 MPa), siendo éste menor en las siguientes (17 MPa). Sin embargo, cabe destacar que el material

Tabla II. Mejora en la dureza (en porcentaje) de muestras procesadas por ECAE

Table II. Increase in hardness (expressed in percentage) of samples processed by ECAE

\section{Estado inicial $\rightarrow$ Estado final}
$\mathrm{N} 0 \rightarrow \mathrm{N} 2$
$\mathrm{N} 0 \rightarrow \mathrm{N} 4$
$\mathrm{NO} \rightarrow \mathrm{N} 14$
$\mathrm{N} 2 \rightarrow \mathrm{N} 4$
$\mathrm{N} 4 \rightarrow \mathrm{N} 14$

\section{Incremento porcentual} de dureza

$[46,3 \pm 3,7] \%(k=2)$

$[56,7 \pm 3,4] \%(k=2)$

$[71,9 \pm 2,8] \%(\mathrm{k}=2)$

$[7,1 \pm 2,8] \%(k=2)$

$[9,7 \pm 1,9] \%(k=2)$ 


\section{Evolución de la Rm y el A\% frente al número de pasadas (N)}

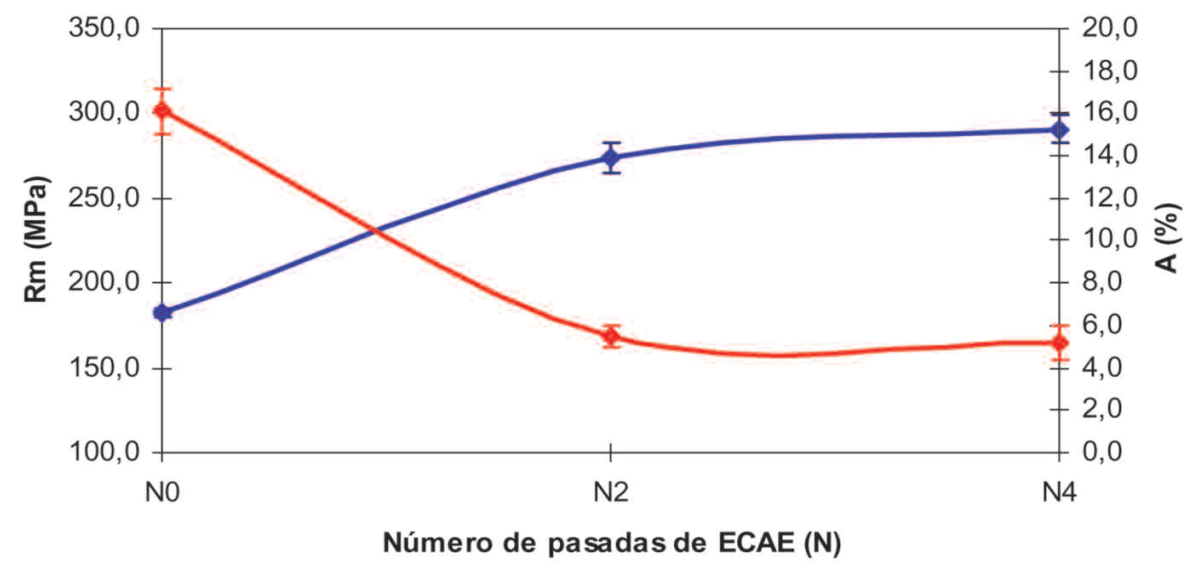

$\leadsto-\operatorname{Rm}(\mathrm{Mpa}) \multimap \mathrm{A}(\%)$

Figura 6. Variación de la resistencia máxima a tracción y del alargamiento a rotura frente al número de pasadas de ECAE de la aleación AA6060.

\section{Figure 6. Variation of the ultimate tensile strength and the elongation to fracture versus number of ECAE passages in the case of AA6060.}

presenta en su estado inicial, una elevada resistencia máxima a tracción, ya que existen precipitados correspondientes a fases intermedias como consecuencia del proceso de fabricación, debido a que como se ha indicado anteriormente, se ha elegido como material inicial la aleación de aluminio AA6060 F. Por lo tanto, la mejora obtenida tras ser procesado por ECAE no es tan acusada como la que se conseguiría si el material no estuviese parcialmente precipitado. Es decir, si se hubiera templado y deformado por ECAE para luego ser sometido a diferentes tratamientos térmicos. Sin embargo, esto tiene el inconveniente de alargar el proceso de fabricación de la aleación a la vez que encarecer los costes.

El alargamiento evoluciona de la misma forma pero en sentido opuesto, produciéndose el principal descenso tras las dos primeras pasadas $(10,6 \%)$ y siendo mucho menor su variación en las siguientes pasadas $(0,5 \%)$. Tras la realización de ensayos con péndulo Charpy, los resultados de energía absorbida por las probetas de $81 \mathrm{~mm}^{2}$ de sección, correspondientes a No, $\mathrm{N} 2$ y N4, fueron de $25 \mathrm{~J}, 17 \mathrm{~J}$ y $15 \mathrm{~J}$, respectivamente, lo que es coherente con la disminución en el alargamiento a rotura experimentada al aumentar el número de pasadas de ECAE.

El comportamiento anteriormente descrito, puede justificarse atendiendo a la energía de deformación acumulada y a la gran densidad de dislocaciones existente en la microestructura de los materiales procesados, generado por el proceso de ECAE, que bloquea los sistemas de deslizamiento, endureciendo el material y reduciendo la capacidad de fluencia del mismo y de ahí que la mejora disminuya al aumentar el número de pasadas de ECAE.

En las primeras pasadas, a medida que se va introduciendo deformación, se producen las principales modificaciones en las propiedades mecánicas hasta que se llega a una situación de saturación de dislocaciones donde ya no se varían significativamente los comportamientos mecánicos del material.

En las figuras 7-11 se muestran varias micrografías obtenidas mediante el empleo de un microscopio óptico

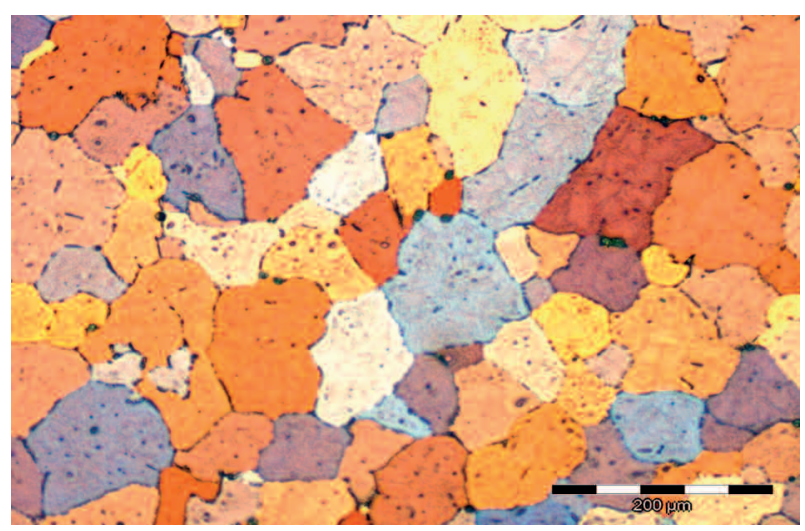

Figura 7. Micrografía óptica de la aleación AA6060 en estado N0, realizada a X100.

Figure 7. Optical micrograph at $X 100$ of $A A 6060$ in NO state. 
con $100 \mathrm{X}$ y $200 \mathrm{X}$ aumentos. En ellas se muestra la microestructura de la aleación AA6060, en tres de los estados de deformación analizados, correspondientes a No, N2 y N4. Se utilizó la técnica de revelado Barker, útil para estudiar la microestructura mediante microscopio óptico y luz polarizada en aleaciones de aluminio.

En la figura 7 se muestran los granos correspondientes a la aleación en estado de partida que tienen un tamaño promedio de aproximadamente $100 \mu \mathrm{m}$. Tal y como se puede apreciar, no aparecen bandas de deformación, por lo que no hay grandes acumulaciones de dislocaciones debido a que no ha sido procesada por ECAE. Se debe indicar que la aleación tras su fabricación contiene ya una determinada densidad de dislocaciones.

En las figuras 8 y 9 , se muestran probetas en estado N2. Se observan claramente bandas de deformación que surgen debido a la alta acumulación de dislocaciones generadas por la deformación plástica severa aplicada. Se aprecia direccionalidad en las bandas, orientadas consecuentemente hacia la dirección de extrusión del material. Debido a que la aleación ha seguido una ruta $\mathrm{C}$, los granos han sido deformados en direcciones perpendiculares, obteniéndose una morfología equiaxial similar a la que poseía el material antes de ser deformado, pero conteniendo un alto número de bandas de deformación.

En las figuras 10 y 11, correspondientes al estado N4, se observan bandas de deformación con mayor densidad que en el caso anterior. Las bandas observadas en las figuras 8-11, son un indicador visual de que existe una densidad muy elevada de dislocaciones en el material.

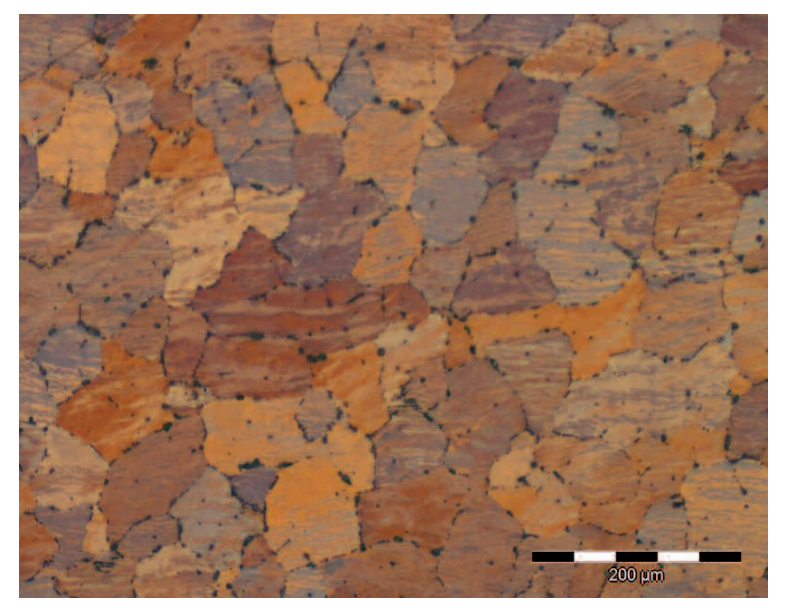

Figura 8. Micrografía óptica de la aleación AA6060 en estado N2, realizada a X100.

Figure 8. Optical micrograph at $X 100$ of AA6060 in N2 state.

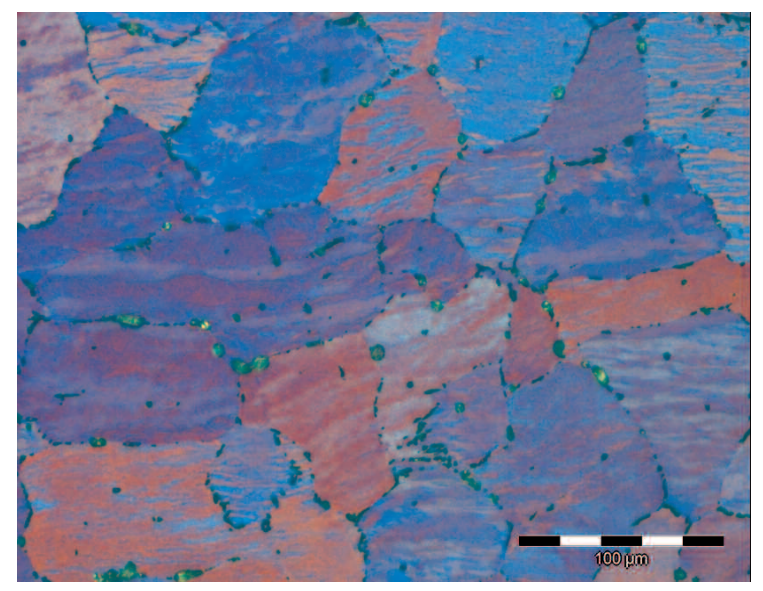

Figura 9. Micrografía óptica de la aleación AA6060 en estado N2, realizada a X200.

Figure 9. Optical micrograph at X200 of AA6060 in N2 state.

En la figura 12, correspondiente al estado N12, se muestra una micrografía realizada con microscopía electrónica de barrido en la que se puede observar tamaño de grano menor a $1 \mu \mathrm{m}$.

\subsection{Análisis de la influencia de los tratamientos de envejecimiento en probetas procesadas por ECAE}

Con el fin de analizar los comportamientos mecánicos de la aleación AA6060 en los distintos estados de deformación No, N2 y N4, frente al fenómeno de

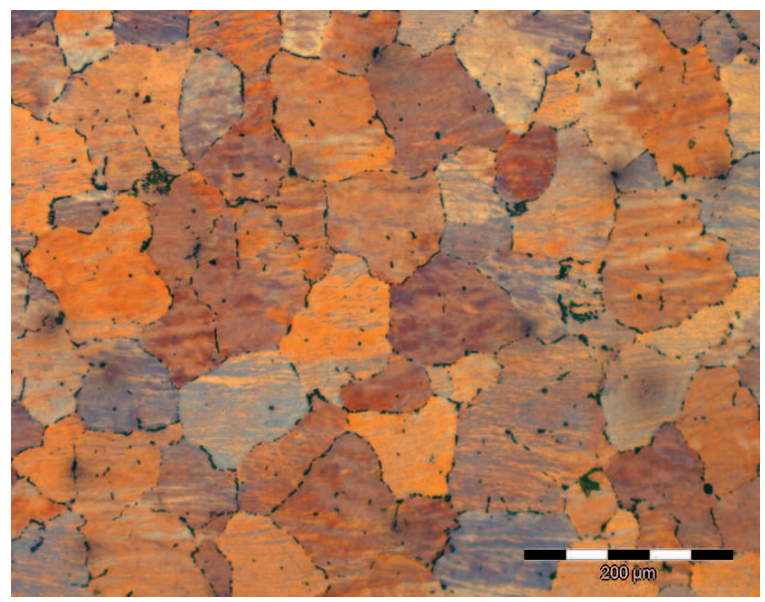

Figura 10. Micrografía óptica de la aleación AA6060 en estado N4, realizada a X100.

Figure 10. Optical micrograph at X100 of AA6060 in N4 state. 


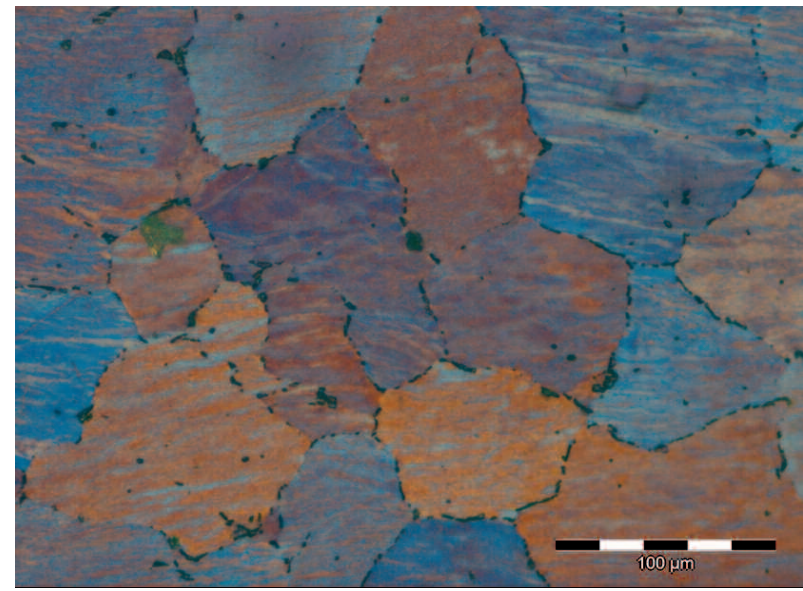

Figura 11. Micrografía óptica de la aleación AA6060 en estado N4, realizada a X200.

Figure 11. Optical micrograph at X200 of AA6060 in N4 state.

envejecimiento, se ha realizado un seguimiento de la dureza, para cada una de las temperaturas de precipitación aplicadas de cara a determinar los tratamientos más adecuados para cada una de las muestras, así como analizar las variaciones en la dureza. Se realizaron para ello diez mediciones de dureza por cada punto representado. Cabe señalar, que únicamente se han considerado N2 y N4 ya que la mejora obtenida a partir de N4 no es significativa, tal y como se ha comentado en el apartado anterior.

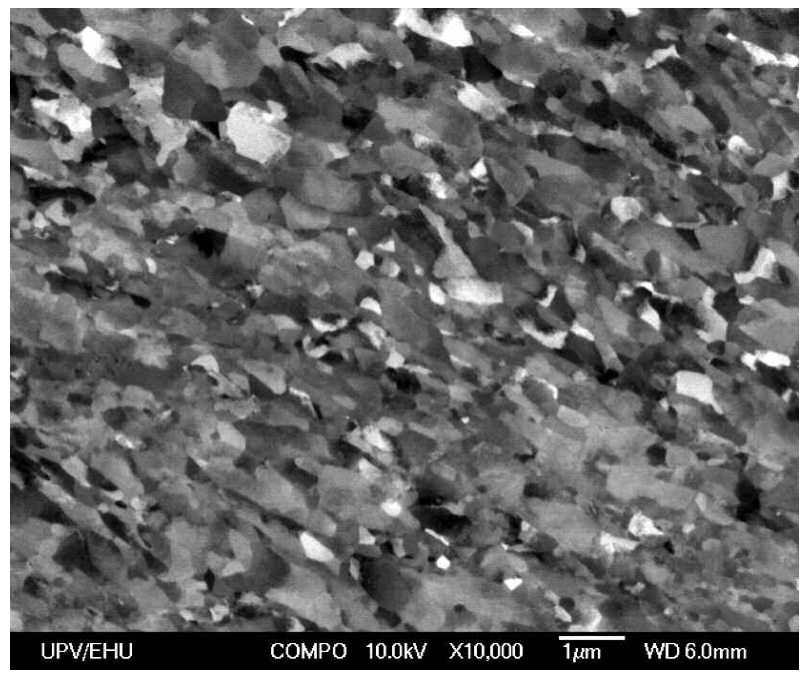

Figura 12. Micrografía electrónica de barrido de la aleación AA6060 en estado N12, realizada a X10000.

Figure 12. SEM micrograph at $X 10000$ of AA6060 in N12 state.

En la figura 13 se representa la variación de la dureza en probetas en estado inicial, al ser aplicados diferentes tratamientos de envejecimiento. Se puede considerar que en el caso mostrado en la figura 13, el envejecimiento es de tipo estático ya que la precipitación de segundas fases tiene lugar sobre una matriz que no ha sido sometida a ningún proceso de

\section{Curva de envejecimiento de la AA6060 No}

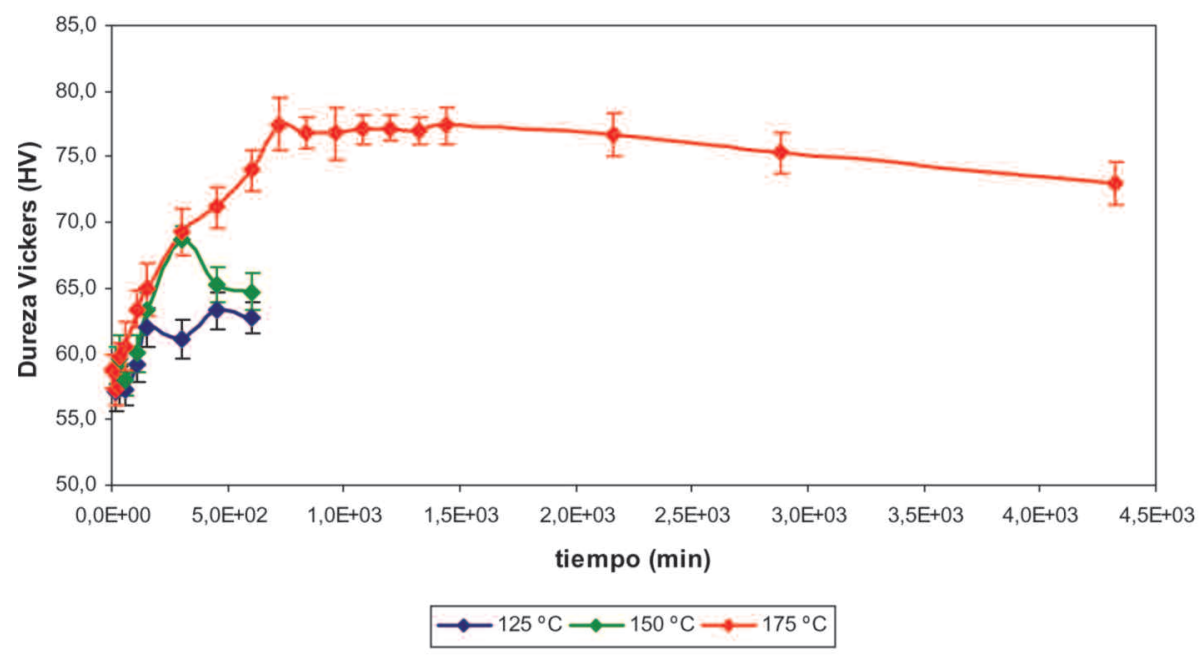

Figura 13. Variación de la dureza de la aleación AA6060 en estado N0 con respecto a los tiempos de tratamiento térmico $\left(125,150\right.$ y $\left.175^{\circ} \mathrm{C}\right)$.

Figure 13. Variation of hardness for AA6060 in NO state with respect to the duration time of heat treatment $\left(125,150\right.$ and $\left.175^{\circ} \mathrm{C}\right)$. 
deformación y, por tanto, contiene una densidad de dislocaciones baja.

Los valores de dureza más elevados se consiguen al aplicar la mayor temperatura estudiada, en este caso a $175^{\circ} \mathrm{C}$. Esto puede ser debido a que la energía aportada, tanto a $125^{\circ} \mathrm{C}$ como a $150^{\circ} \mathrm{C}$, no es suficiente para que la mayor parte de los aleantes que se encuentran en disolución formen compuestos y precipiten en la matriz, bloqueando los planos de deslizamiento y endureciendo el material.

Cuando se aplica la temperatura de $175^{\circ} \mathrm{C}$ a la probeta en estado $\mathrm{NO}$, se encuentra un valor máximo de la dureza correspondiente a $12 \mathrm{~h}$ de tratamiento de 77,5 HV, como se puede observar en la figura 13. Este pico de dureza puede deberse a los mecanismos de precipitación implicados en esta aleación, justificándose este hecho con las distintas geometrías y cinéticas de los precipitados de $\mathrm{Mg}_{2} \mathrm{Si}$ y las transformaciones existentes entre ellos, correspondiendo a zonas Guinier-Preston y a fases $\theta^{\prime \prime}, \theta^{\prime}$ y $\theta\left(\mathrm{Mg}_{2} \mathrm{Si}\right)$, según el estudio realizado por Roven ${ }^{[11]}$. Estas zonas y fases pueden ser coherentes, semicoherentes o incoherentes con los planos característicos de la matriz.

Para el caso del tratamiento a $150^{\circ} \mathrm{C}$, tal y como se observa en la figura 13, el máximo corresponde a un tiempo de tratamiento térmico de $5 \mathrm{~h}$. Cuando se aplica al material la temperatura de $125^{\circ} \mathrm{C}$, no se observan claramente máximos en los valores de dureza.

Sin embargo, a $125^{\circ} \mathrm{C}$ es posible que no se haya aportado la energía suficiente para haber precipitado totalmente las fases que se encuentran disueltas en la matriz. Por ello, el valor de dureza obtenido al cabo de $10 \mathrm{~h}$ es menor que en los casos anteriores.

Por lo tanto, se escoge la temperatura de $175^{\circ} \mathrm{C}$ como aquella que ofrece los resultados más elevados de dureza, completándose el estudio mediante la realización de ensayos de tracción.

A continuación, se cuantifican los incrementos porcentuales de dureza obtenidos mediante el envejecimiento utilizando la ecuación (4), donde $\mathrm{HVtf}$ y $\mathrm{HVti}$, son los valores de dureza a tiempo final e inicial de tratamiento, respectivamente.

$$
\frac{H V t f-H V t i}{H V t i} * 100
$$

Se estudian cuatro puntos característicos correspondientes a tiempos de $105 \mathrm{~min}(1,45 \mathrm{~h}), 5,10 \mathrm{y}$ $12 \mathrm{~h}$ de tratamiento. Los porcentajes de mejora se expresan en la tabla III.

Tal y como se pone de manifiesto a partir de los resultados de la tabla III, se producen mejoras en la dureza. Así, los mejores tratamientos, atendiendo a la dureza, son los correspondientes a los tiempos de tratamiento de $5 \mathrm{~h}$ y $12 \mathrm{~h}$, con mejoras del $18,1 \%$ y
Tabla III. Mejora en la dureza (en porcentaje) en muestras en estado N0 con envejecimiento posterior

\section{Table III. Increase in hardness (expressed in percentage) of samples in NO state with subsequent aging}

$\begin{array}{cc}\text { Estado inicial } \rightarrow & \begin{array}{c}\text { Incremento porcentual } \\ \text { Estado final }\end{array} \\ \text { de dureza }\end{array}$

$\begin{array}{lr}\mathrm{N} 0 \rightarrow \mathrm{N} 0 \mathrm{t}=1,45 \mathrm{~h} & {[8,1 \pm 2,2] \%(\mathrm{k}=2)} \\ \mathrm{N} 0 \rightarrow \mathrm{N} 0 \mathrm{t}=5 \mathrm{~h} & {[18,1 \pm 2,6] \%(\mathrm{k}=2)} \\ \mathrm{N} 0 \rightarrow \mathrm{N} 0 \mathrm{t}=10 \mathrm{~h} & {[26,1 \pm 2,5] \%(\mathrm{k}=2)} \\ \mathrm{N} 0 \rightarrow \mathrm{N} 0 \mathrm{t}=12 \mathrm{~h} & {[32,1 \pm 2,9] \%(\mathrm{k}=2)}\end{array}$

$32,1 \%$, respectivamente. A continuación, se analiza el comportamiento de la resistencia mecánica y del alargamiento por medio de ensayos de tracción por triplicado, con objeto de conocer con mayor profundidad el comportamiento mecánico del material.

En la figura 14 se representa la evolución de la resistencia máxima a tracción y del alargamiento a rotura de las probetas en estado No, frente al tiempo. La resistencia máxima a tracción evoluciona de forma similar a la dureza. Atendiendo al alargamiento a rotura experimentado por el material, se puede comprobar que va disminuyendo conforme aumenta el tiempo de tratamiento. Esto puede ser debido al bloqueo de los planos de deslizamiento provocado por las segundas fases que han precipitado y por la morfología que presentan en ese momento.

A continuación, se aplica el mismo razonamiento para estudiar las probetas procesadas por ECAE. En estos casos de $\mathrm{N} 2$ y N4, la precipitación se produce sobre una matriz en la que se ha introducido una elevada densidad de deformación, por ello, las dislocaciones existentes son zonas de energía que utilizan las segundas fases en disolución para poder precipitar en forma de compuestos. Este hecho provoca que se produzca una precipitación fina y dispersa, como se puede observar en estudios como el de Kim y Wang ${ }^{[14]}$, que en combinación con la alta densidad de dislocaciones introducidas en el material, proporciona una mejora en las propiedades mecánicas, como la dureza o la resistencia mecánica.

En las figuras 15 y 16, se muestra la modificación de la dureza de las probetas en estado $\mathrm{N} 2$ y N4, respectivamente, para cada una de las tres temperaturas de envejecimiento analizadas.

En la figura 15 y en la figura 16 se pone de manifiesto que los valores de dureza más elevados se obtienen al aplicar la menor temperatura, en este caso $125^{\circ} \mathrm{C}$. 


\section{Evolución de la $\mathrm{Rm}$ y el $\mathrm{A} \%$ frente a tiempo de la AA6060 N0 a T175 ${ }^{\circ} \mathrm{C}$}

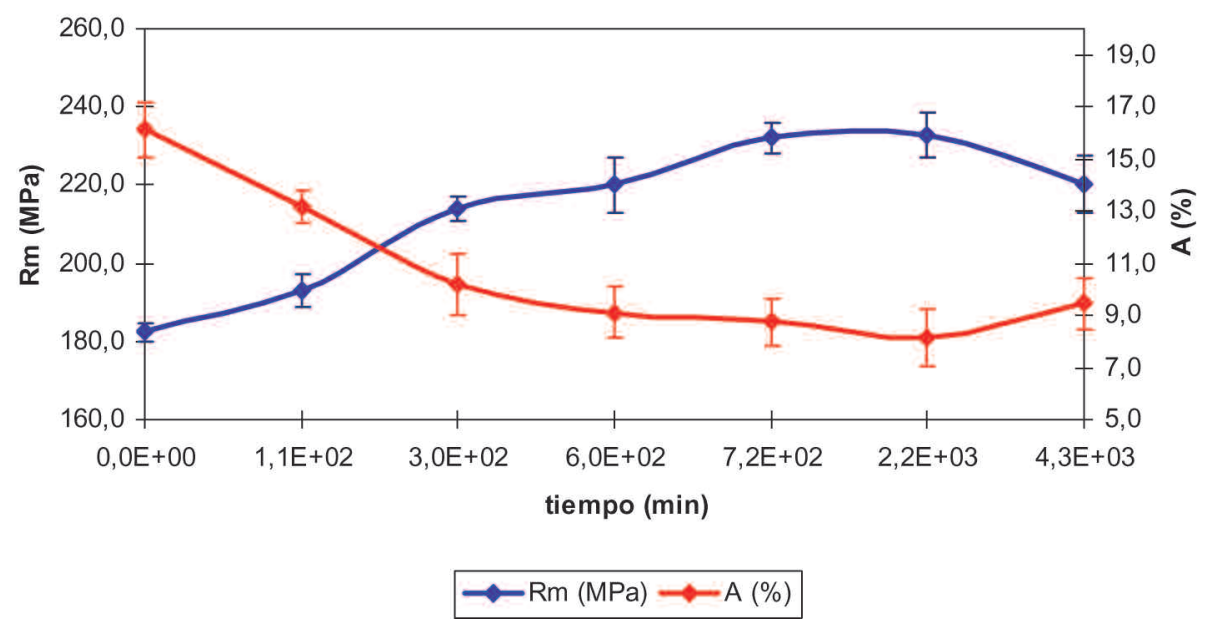

Figura 14. Variación de la resistencia máxima a tracción y del alargamiento a rotura de la aleación AA6060 en N0 respecto al tiempo de tratamiento térmico realizado a $175^{\circ} \mathrm{C}$.

Figure 14. Variation of the ultimate tensile strength and the elongation to fracture for AA6060 in NO state with respect to the duration time of the heat treatment performed at $175^{\circ} \mathrm{C}$.

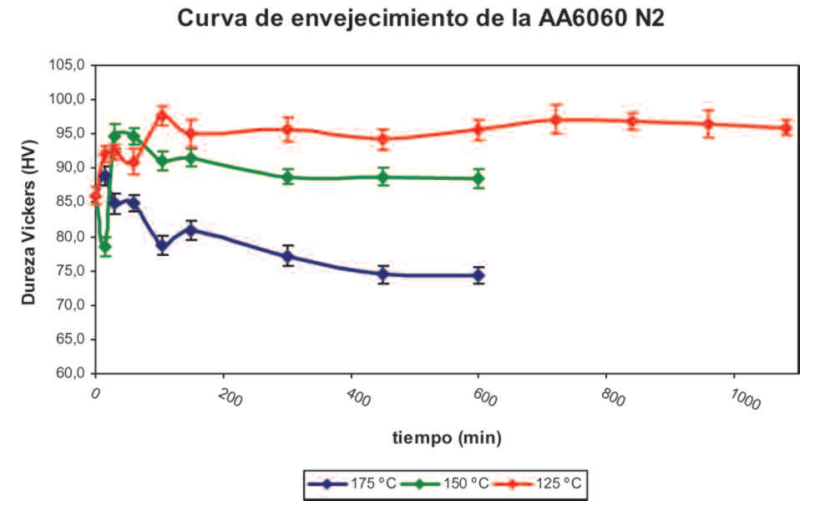

Figura 15. Variación de la dureza de la aleación AA6060 en estado N2 con respecto a los tiempos de tratamiento térmico $\left(125,150\right.$ y $\left.175^{\circ} \mathrm{C}\right)$

Figure 15. Variation of hardness for $A A 6060$ in N2 state with respect to the duration time of heat treatment $\left(125,150\right.$ and $\left.175^{\circ} \mathrm{C}\right)$.

Roven ${ }^{[11]}$ propone como principales razones del endurecimiento la interacción cooperativa existente entre los precipitados finos de $\theta$ "'- $\mathrm{Mg}_{2} \mathrm{Si}$ y la alta densidad de dislocaciones introducidas mediante ECAE. El afinamiento de grano puede ser otro de los motivos que modifican esta propiedad mecánica. Sin embargo, en el presente estudio no se analizará este

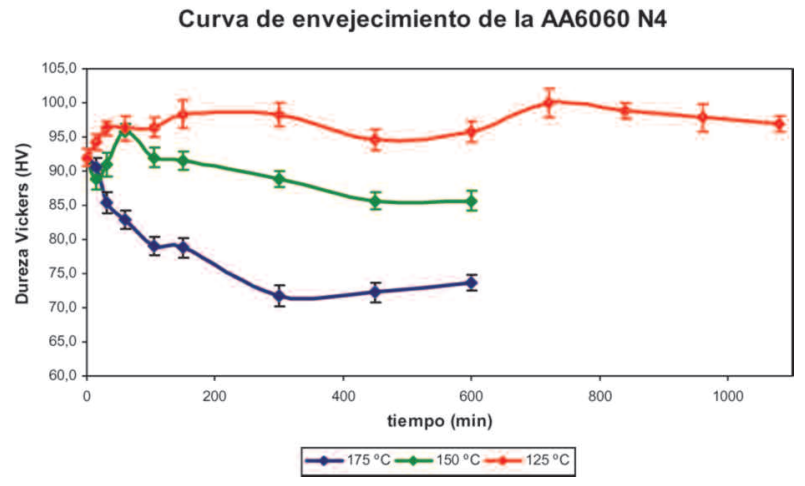

Figura 16. Variación de la dureza de la aleación AA6060 en estado N4 con respecto a los tiempos de tratamiento térmico $\left(125,150\right.$ y $\left.175^{\circ} \mathrm{C}\right)$

Figure 16. Variation of hardness for $A A 6060$ in N4 state with respect to the duration time of heat treatment $\left(125,150\right.$ and $\left.175^{\circ} \mathrm{C}\right)$.

aspecto. Atendiendo a este hecho, es posible que los bajos valores de dureza obtenidos al aplicar las mayores temperaturas de envejecimiento puedan ser atribuidos tanto a la aniquilación parcial de las dislocaciones, previamente introducidas por ECAE, como a la precipitación y crecimiento de fases de $\mathrm{Mg}_{2} \mathrm{Si}$, con morfologías incoherentes con la matriz. Dichas 
fases son incapaces de bloquear efectivamente los planos de deslizamiento debido al exceso de energía proporcionado al material. Este hecho se intensifica conforme se eleva la temperatura, como se puede apreciar en las figuras 15 y 16.

En N2 y en N4, no existe convergencia en los valores de dureza en los tratamientos de $12 \mathrm{~h}$. Esto puede ser debido a que las distintas morfologías de los precipitados afectan seriamente a las propiedades del material. Además, la aniquilación de dislocaciones aumentará con la temperatura aplicada en cada caso.

Por lo tanto, tras haber analizado el estudio de envejecimiento dinámico en probetas en estado $\mathrm{N} 2$ y N4, se escogerá la temperatura de $125^{\circ} \mathrm{C}$ para determinar algunas propiedades mecánicas más mediante la realización de ensayos de tracción.

En la figura 15 se muestra el estudio de la dureza de probetas en estado N2 en función del tiempo de tratamiento. Como se puede observar, surgen unos leves picos a $1,45 \mathrm{~h}$, a $5 \mathrm{~h}$ y a $12 \mathrm{~h}$ de tratamiento, aunque podrían ser poco significativos si se tiene en cuenta en cuenta la incertidumbre de medida del equipo y el número de medidas realizadas (entre 0,5 y 2 HV). Utilizando nuevamente la ecuación (4), se analiza la mejora obtenida en esta propiedad en cada uno de estos tres puntos considerados.

Tal y como se puede comprobar en la tabla IV, se consiguen mejoras notables en la dureza del material, incrementándose más de un $10 \%$ respecto a las pro-
Tabla IV. Mejora en la dureza (en porcentaje) en muestras procesadas por ECAE hasta N2 con envejecimiento posterior

Table IV. Increase in hardness (expressed in percentage) of samples in N2 state with subsequent aging

$\begin{array}{cc}\text { Estado inicial } \rightarrow & \begin{array}{c}\text { Incremento porcentual } \\ \text { Estado final }\end{array} \\ \text { de dureza }\end{array}$

$\begin{array}{ll}\mathrm{N} 2 \rightarrow \mathrm{N} 2 \mathrm{t}=1,45 \mathrm{~h} & {[13,6 \pm 2,7] \%(\mathrm{k}=2)} \\ \mathrm{N} 2 \rightarrow \mathrm{N} 2 \mathrm{t}=5 \mathrm{~h} & {[11,4 \pm 2,4] \%(\mathrm{k}=2)} \\ \mathrm{N} 2 \rightarrow \mathrm{N} 2 \mathrm{t}=10 \mathrm{~h} & {[11,3 \pm 2,5] \%(\mathrm{k}=2)} \\ \mathrm{N} 2 \rightarrow \mathrm{N} 2 \mathrm{t}=12 \mathrm{~h} & {[13,0 \pm 2,6] \%(\mathrm{k}=2)}\end{array}$

betas procesadas por ECAE, sin tratamiento posterior de envejecimiento

En la figura 17 se puede comprobar que la resistencia máxima a tracción evoluciona de forma similar a la dureza representada en la figura 15 .

$\mathrm{Al}$ contrario de lo que ocurre con las probetas en estado inicial, el alargamiento a rotura va aumentando conforme se incrementa la duración del tratamiento. Este hecho puede justificarse en base a la existencia de tres fenómenos distintos que interfieren entre ellos. El primero es que los precipitados existentes, además de endurecer el material por blo-

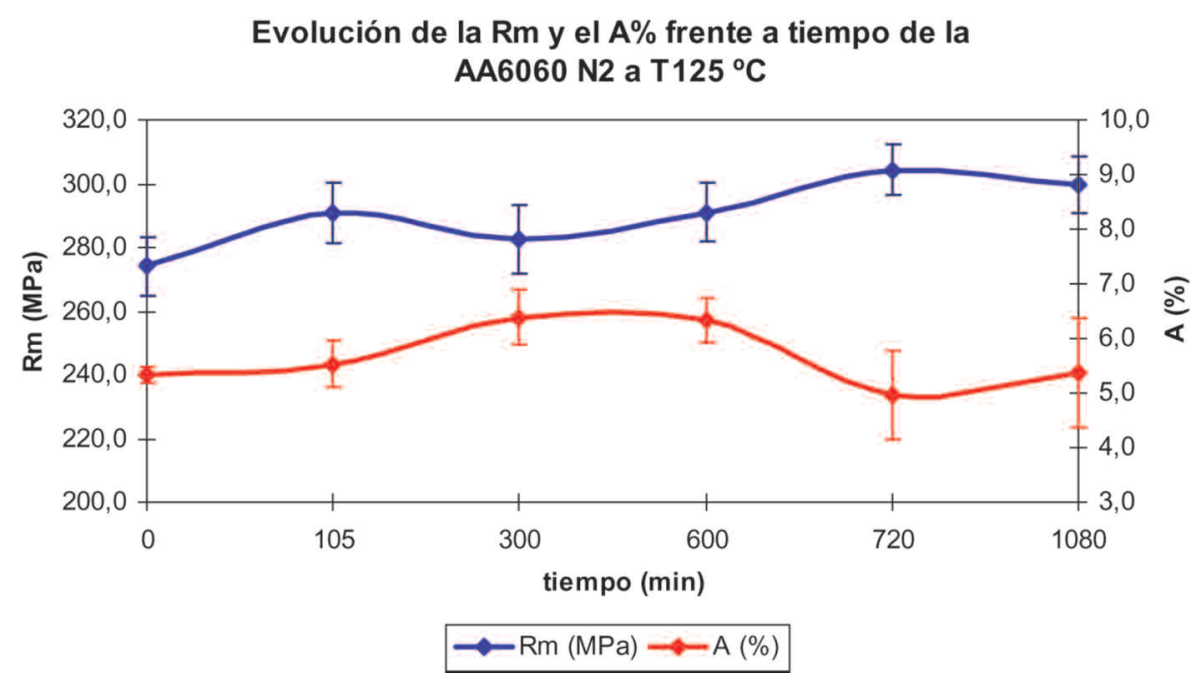

Figura 17. Variación de la resistencia máxima a tracción y del alargamiento a rotura de la aleación AA6060 en N2 con respecto al tiempo de tratamiento térmico realizado a $125^{\circ} \mathrm{C}$.

Figure 17. Variation of the ultimate tensile strength and the elongation to fracture for AA6060 in N2 state with respect to the duration time of the heat treatment performed at $125^{\circ} \mathrm{C}$. 
queo de dislocaciones, sean lo suficientemente finos como para no comprometer en gran medida el alargamiento a rotura del material. El segundo efecto que puede estar presente, es el alivio de tensiones del material, que puede mejorar el alargamiento sin provocar grandes cambios en la resistencia mecánica. En tercer lugar, la aniquilación de dislocaciones y la consecuente movilidad de los planos de deslizamiento favorecen la fluencia del material y aunque conlleva una disminución de la dureza ésta puede ser contrarrestada por el efecto endurecedor de los precipitados presentes.

Se puede concluir, que el tiempo de tratamiento óptimo para conseguir probetas con buenas propiedades mecánicas es el correspondiente a $5 \mathrm{~h}$, como se muestra en la figura 17. En la tabla $\mathrm{V}$ se representan las mejoras en dureza y alargamiento a rotura en probetas en estado $\mathrm{N} 2$ con tratamiento térmico posterior.

Análogamente al estudio realizado para las probetas en estado de deformación N2 y atendiendo a la figura 16 , se obtienen los incrementos de dureza, correspondiente a los tiempos de tratamiento a $1,45 \mathrm{~h}, 5 \mathrm{~h}, 10 \mathrm{~h}$ y $12 \mathrm{~h}$ en muestras procesadas por ECAE hasta N4.

Como se puede apreciar en la tabla VI, la mejora obtenida en probetas en estado N4 es menor a las conseguidas en NO ó N2. Este hecho puede estar relacionado con que el efecto endurecedor de la precipitación no sea capaz de contrarrestar la pérdida de dureza debido al alivio de las elevadas deformaciones que contiene el material.

En la figura 18 se muestran los resultados obtenidos mediante los ensayos de tracción. Si bien la resistencia máxima a tracción muestra una evolución similar a la dureza, el alargamiento a rotura se incrementa a medida que aumenta el tiempo de envejecimiento. En este hecho, nuevamente están implicados los mismos fenómenos detallados anteriormente para el caso de las probetas en estado N2. La restauración puede contribuir a la creación de celdas, mediante la difusión de dislocaciones que proporcionan un mayor alargamiento a rotura al mate-
Tabla VI. Mejora en la dureza (en porcentaje) en muestras procesadas por ECAE hasta N4 con envejecimiento posterior
Table VI. Increase in hardness (expressed in percentage) of samples in N4 state with subsequent aging

\section{Estado inicial $\rightarrow$ Estado final \\ Incremento porcentual de dureza}

$\begin{array}{ll}\mathrm{N} 4 \rightarrow \mathrm{N} 4 \mathrm{t}=1,45 \mathrm{~h} & {[4,8 \pm 1,8] \%(\mathrm{k}=2)} \\ \mathrm{N} 4 \rightarrow \mathrm{N} 4 \mathrm{t}=5 \mathrm{~h} & {[6,8 \pm 2,0] \%(\mathrm{k}=2)} \\ \mathrm{N} 4 \rightarrow \mathrm{N} 4 \mathrm{t}=10 \mathrm{~h} & {[4,1 \pm 1,8] \%(\mathrm{k}=2)} \\ \mathrm{N} 4 \rightarrow \mathrm{N} 4 \mathrm{t}=12 \mathrm{~h} & {[8,7 \pm 2,1] \%(\mathrm{k}=2)}\end{array}$

rial. La posible precipitación fina junto con un cierto alivio de tensiones por medio de la aniquilación parcial de dislocaciones son efectos que tienden a mejorar el alargamiento del material, tal y como se muestra en el trabajo de Roven ${ }^{[11]}$.

Al mantenerse la resistencia máxima a tracción y la dureza con pocas variaciones a lo largo del tratamiento, se escoge como punto óptimo de tratamiento aquel que mayor alargamiento a rotura experimenta, correspondiente a $5 \mathrm{~h}$ de envejecimiento.

Como se observa en la tabla VII, el alargamiento a rotura de las probetas en $\mathrm{N} 4$ tras los tratamientos de envejecimiento, aumenta un $30 \%$ aproximadamente, respecto a las probetas en N4 sin tratamientos de precipitación posterior.

\section{CONCLUSIONES}

- Se ha observado que el proceso de deformación plástica severa de ECAE, produce notables incrementos de dureza y resistencia máxima a tracción en el material. Dicho aumento se produce prin-

Tabla V. Mejora en la dureza y en el alargamiento a rotura, en muestras N2 con envejecimiento posterior

Table V. Increases in hardness and elongation to fracture (expressed in percentage) of samples in N2 state with subsequent aging

\begin{tabular}{lcc}
\hline $\begin{array}{c}\text { Estado inicial } \rightarrow \\
\text { Estado final }\end{array}$ & Dureza (\%) & $\begin{array}{c}\text { Alargamiento a } \\
\text { Rotura (\%) }\end{array}$ \\
\hline $\mathrm{N} 2 \rightarrow \mathrm{N} 2 \mathrm{t}=5 \mathrm{~h}$ & {$[11,4 \pm 2,4] \%(\mathrm{k}=2)$} & {$[20,8 \pm 0,3] \%(\mathrm{k}=3)$} \\
$\mathrm{N} 2 \rightarrow \mathrm{N} 2 \mathrm{t}=10 \mathrm{~h}$ & {$[11,3 \pm 2,5] \%(\mathrm{k}=2)$} & {$[22,6 \pm 0,3] \%(\mathrm{k}=3)$} \\
$\mathrm{N} 2 \rightarrow \mathrm{N} 2 \mathrm{t}=12 \mathrm{~h}$ & {$[13,0 \pm 2,6] \%(\mathrm{k}=2)$} & {$[-7,1 \pm 0,3] \%(\mathrm{k}=3)$} \\
\hline
\end{tabular}




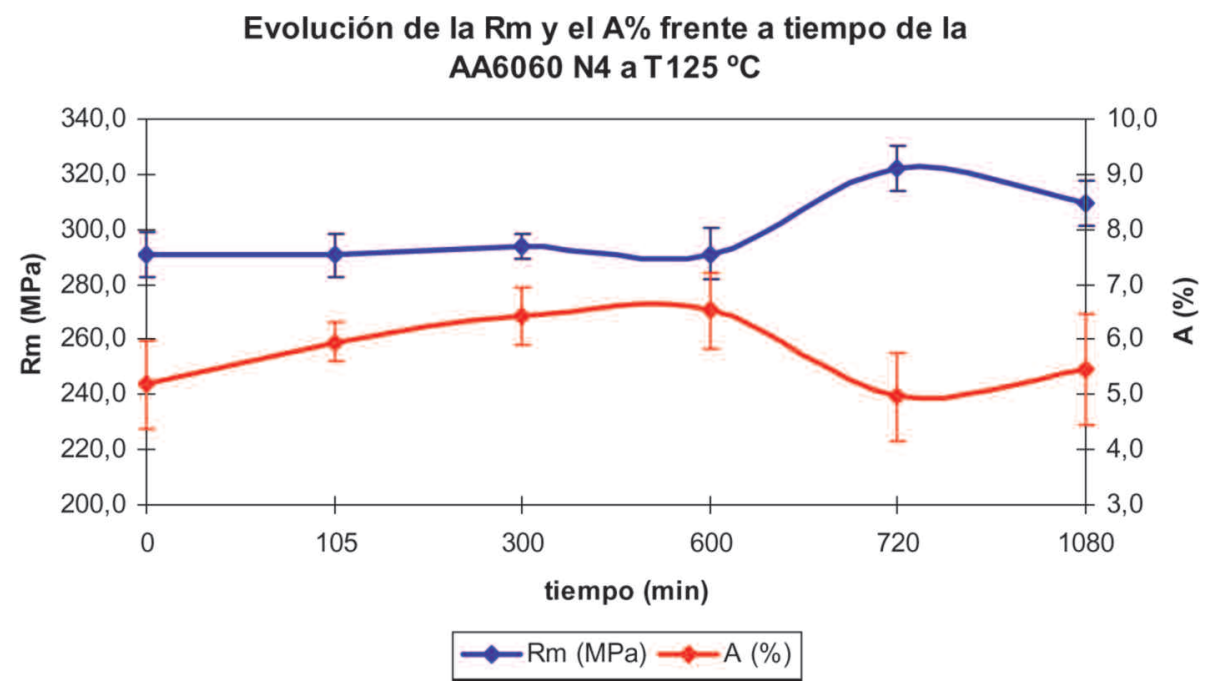

Figura 18. Variación de la resistencia máxima a tracción y del alargamiento a rotura de la aleación AA6060 en N4 con respecto al tiempo de tratamiento térmico realizado a $125^{\circ} \mathrm{C}$.

Figure 18. Variation of the ultimate tensile strength and the elongation to fracture for AA6060 in N4 state with respect to the duration time of the heat treatment performed at $125^{\circ} \mathrm{C}$.

Tabla VII. Mejora en la dureza y en el alargamiento a rotura, en muestras N4 con envejecimiento posterior

Table VII. Increases in hardness and elongation to fracture (expressed in percentage) of samples in N4 state with subsequent aging

\begin{tabular}{lcc}
\hline $\begin{array}{c}\text { Estado inicial } \rightarrow \\
\text { Estado final }\end{array}$ & Dureza (\%) & $\begin{array}{c}\text { Alargamiento a } \\
\text { Rotura (\%) }\end{array}$ \\
\hline $\mathrm{N} 4 \rightarrow \mathrm{N} 4 \mathrm{t}=5 \mathrm{~h}$ & {$[6,8 \pm 2,0] \%(\mathrm{k}=2)$} & {$[28,8 \pm 0,4] \%(\mathrm{k}=3)$} \\
$\mathrm{N} 4 \rightarrow \mathrm{N} 4 \mathrm{t}=10 \mathrm{~h}$ & {$[4,1 \pm 1,8] \%(\mathrm{k}=2)$} & {$[32,7 \pm 0,5] \%(\mathrm{k}=3)$} \\
$\mathrm{N} 4 \rightarrow \mathrm{N} 4 \mathrm{t}=12 \mathrm{~h}$ & {$[8,7 \pm 2,1] \%(\mathrm{k}=2)$} & {$[-4,2 \pm 0,4] \%(\mathrm{k}=3)$} \\
\hline
\end{tabular}

cipalmente en las dos primeras pasadas, perdiendo efectividad al aumentar el número de pasadas como consecuencia de la acumulación de deformación plástica en el material procesado.

- Tras realizar los estudios de envejecimiento sin deformación previa en probetas en estado de partida se pusieron de manifiesto los siguientes hechos:

- La temperatura de tratamiento para la que se obtuvieron mejores comportamientos mecánicos fue la de $175^{\circ} \mathrm{C}$. Es posible que la energía aplicada en los tratamientos de precipitación de menor temperatura no sea suficiente para provocar la completa difusión de precipitados intermedios y por tanto la precipitación total. En el tratamiento escogido como óptimo $\left(175^{\circ} \mathrm{C}\right.$ durante $\left.12 \mathrm{~h}\right)$, se obtuvo una mejora en la dureza del $32,1 \%$ y una pérdida de alargamiento a rotura de un 7,3\% respecto al material en estado de partida.

- Tras realizar el envejecimiento con deformación plástica severa previa en probetas en estado N2 y $\mathrm{N} 4$, se obtuvieron las siguientes conclusiones:

- La temperatura de $125^{\circ} \mathrm{C}$ es la que proporciona mejores resultados en el comportamiento mecánico. El hecho de que la dureza aumente y el alargamiento a rotura disminuya de manera poco significativa puede ser debido a que la restauración y la consecuente aniquilación de dislocacio- 
nes incrementan la fluencia del material y aunque este hecho conlleve una consecuente pérdida de resistencia mecánica del material, ésta se vea compensada por el efecto endurecedor proporcionado por la precipitación de precipitados intermedios. Si se aplica un tratamiento que aporte mayor energía, es posible que el alivio de tensiones provoque una pérdida de dureza demasiado elevada para ser compensada por el envejecimiento o, incluso, provocar el sobreenvejecimiento del material. El tratamiento escogido como óptimo $\left(125^{\circ} \mathrm{C}\right.$ y $\left.12 \mathrm{~h}\right)$ proporcionó una mejora del 13,0 \% de dureza y una leve disminución del alargamiento a rotura en probetas en estado N2 y respectivamente un aumento del 8,7 \% de dureza y una pequeña disminución del alargamiento a rotura en muestras procesadas hasta $\mathrm{N} 4$.

\section{Agradecimientos}

Los autores del presente estudio agradecen el apoyodado por el Ministerio de Ciencia e Innovación (Proyecto de investigación MAT 2006-14341-C02-02).

\section{REFERENCIAS}

[1] M. Furukawa, Z. Horita, M. Nemoto, R.Z. Valiev y T. Langdon, Mater. Charact. 37 (1996) 277-283.

[2] R.Z. Valiev, A.V. Korznikov y R.R. Mulyukov, Mater. Sci. Eng. A 168 (1993)141-148.

[3] V.M. Segal, Russ. Metall. 1 (1981) 99-105.

[4] P.A. Gonzalez, C. Luis-Pérez, Y. Garcés y J. Gil-Sevillano, Rev. Metal. Madrid 37 (2001) 673-692.

[5] M. Hockauf, L.W. Meyer, B. Zillmann, M. Hietschold, S. Schulze y L. Krüger, Mater. Sci. Eng. A, 503 (2009)167-171.

[6] M.J. O'Brien, H.F. Von Bremen, M. Furukawa, Z. Horita y T. Langdon, Mater. Sci. Eng. A, 456 (2007) 236-242.
[7] A.S.M. Agena, J. Mater. Process. Technol. 209 (2009) 856-863.

[8] P.K. Chaudhury, B. Cherukuri y R.Srinivasan, Mater. Sci. Eng. A, 410-411 (2005) 316-318.

[9] S.N. Mathaudhu, A.J. de Rosset, K.T. Hartwig y L.J. Kecskes, Mater. Sci. Eng. A, 503 (2009) 28-31.

[10] N. Lugo, J.M. Cabrera, N. Llorca, C.J. Luis, R. Luri, J. León y I. Puertas, Mater. Sci. Forum 584-586 (2008) 393-398.

[11] H.J. Roven, M. Liu y J.C. Werenskiold, Mater. Sci. Eng. A, 483-484 (2008) 54-58.

[12] M. Ravi Shankar, S. Chandrasekar, A.H. King and W.D. Compton, Acta Mater. 53 (2005) 4.781-4.793.

[13] J.K. Kim, H.G. Jeong, S.I. Hong, Y.S. Kim y W.J. Kim, Scripta Mater. 45-8 (2001) 901-907.

[14] W.J. Kim y J.Y. Wang, Mater. Sci. Eng. A, 464 (2007) 23-27.

[15] UNE 38337:2001, Aluminio y aleaciones de aluminio para forja. Serie 6000. AlMgSi. Aleación EN AW-6063/EN AW-AlMg0,7Si, Ed. AENOR, 2001.

[16] C.J. Luis Pérez, Scripta Mater. 50 (2004) 387-393.

[17] R. Luri, C.J. Luis, J. León y M.A. Sebastian, ASME, Journal of Manufacturing Science and Engineering, Vol. 128, 2006, pp. 860-865.

[18] C.J. Luis Pérez y R. Luri, Mech. Mater. 40 (2008) 617-628.

[19] F.J. Humphreys y M. Hatherly, Recrystallization and Related Annealing Phenomena, Elsevier, Second Edition, Oxford, England, 2004, pp. 451-458.

[20] J. Gil Sevillano, P. Van Houtte y E. Aernoudt, Prog. Mater. Sci. 25 (1980) 69-134.

[21] ISO/IEC Guide 98-3:2008 (Revision of ISO/IEC Guide 98:1995), Guide to the Expression of Uncertainty in Measurement (GUM), 2008, International Organization for Standardization (ISO). 